\title{
DIE SO GENANNTEN SEPOLCRI VON MARC'ANTONIO ZIANI
}

\section{Einleitung}

Das Wiener „Sepolcro“ dient als Gattungsbezeichnung für ein geistlich-dramatisches Vokalwerk, das zwischen 1660 und 1705 und in veränderter Form bis etwa zum Tod Karls VI. in Wien in der Karwoche aufgeführt wurde. Der Begriff leitet sich von zeitgenössischen Quellen ab, welche die Kompositionen bis 1705 vorrangig als Rappresentatione sacra al Santissimo Sepolcro bezeichnen, während sie später diverse Titelzusätze tragen, etwa Componimento sacro, Azione sacra oder auch Oratorio, häufig mit dem Zusatz al SS: Sepolcro.

Bereits mehrfach stand das Sepolcro im Fokus der Musikwissenschaft, wobei das besondere Augenmerk deren Entstehung und Frühzeit galt. ${ }^{1}$ Die vorliegende Studie ist daher jener Zeit gewidmet, in der sich das Sepolcro entscheidend veränderte, sich dem Oratorium anglich, vielleicht in dieses mündete.

Marc'Antonio Ziani wurde um 1652 vermutlich in Venedig geboren. ${ }^{2}$ In den ersten knapp 50 Jahren seines Lebens trat er hauptsächlich als Opernkomponist in Italien in Erscheinung. 1700 wurde er Vizehofkapellmeister von Kaiser Leopold I. und blieb dies unter Joseph I. bis ihn Karl VI. 1712 zum Hofkapellmeister beförderte. Am 22. Jänner 1715 verstarb Ziani in Wien.

Von 1701 bis 1711 komponierte er jährlich ein Sepolcro, wobei das erste nach seinem Tod 1715 noch einmal aufgeführt wurde. Damit widmete er sich diesem Genre unmittelbar nach dem Tod der beiden Protagonisten von dessen Blütezeit: 1698 verstarb Nicolò Minato, der einen großen Teil jener Libretti verfasst hatte, die der 1700 verstorbene Antonio Draghi vertonte.

1 Etwa GRUBER, Gernot. Das Wiener Sepolcro und Johann Joseph Fux: Nach einem Vortrag gehalten vor der Jahreshauptversammlung der Johann-Joseph-Fux-Gesellschaft am 3. Dezember 1968, Graz, 1972. graphie siehe PROMINCZEL, Johannes. Die Kirchenmusik von Marc'Antonio Ziani: Quellen-Analyse - Werkverzeichnis (Dissertation). Wien, 2012, S. 22-29. 


\section{Die Quellen}

Zianis Sepolcri sind uns durch drei verschiedene Quellenarten überliefert: ${ }^{3}$

\section{1) Librettodrucke}

Libretti von sieben Sepolcri Zianis blieben sowohl auf Italienisch, als auch in der zeitgenössischen deutschen Übersetzung erhalten, wobei es sich eher um eine Nachdichtung als um eine wortwörtliche Übertragung handelt.

Von La Tempesta de' dolori (1703) gibt es nur noch das italienische Libretto, ebenso von La sapienza umana (1710) und Il sepolcro nell'orto (1711). Die beiden letzteren werden in der Biblioteca nazionale Marciana in Venedig (I-Vnm) aufbewahrt.

\section{2) Partituren}

Als autographe Partitur blieb - wiederum in der Biblioteca nazionale Marciana - Le due Passioni (1705) erhalten. ${ }^{4}$ Bis auf die ersten drei Werke, von denen die Musik verschollen ist, gibt es zu den übrigen Sepolcri von Kopisten angefertigte Partituren aus dem Bestand der Wiener Hofkapelle.

\section{3) Stimmsätze}

Instrumentalstimmen sind nur von den Werken von 1709 und 1711 überliefert. Bei diesen Stimmsätzen handelt es sich ausschließlich um zwei Violinen, Viola und Violoncello.

\begin{tabular}{|l|l|c|c|c|c|c|}
\hline Kurztitel & Librettist & Jahr & Libretto ita. & Libretto dt. & Partitur & Stimmen \\
\hline $\begin{array}{l}\text { II fascietto di } \\
\text { mirra }\end{array}$ & $\begin{array}{l}\text { Donato } \\
\text { Cupeda? }\end{array}$ & $\begin{array}{l}1701 \\
1715\end{array}$ & $\begin{array}{c}\text { A-Wn 406.742-B.M } \\
(30)\end{array}$ & $\begin{array}{c}\text { A-Wn 406.742-B.M } \\
(31)\end{array}$ & - & - \\
\hline $\begin{array}{l}\text { Le profezie } \\
\text { adempiute }\end{array}$ & $\begin{array}{l}\text { Donato } \\
\text { Cupeda? }\end{array}$ & 1702 & $\begin{array}{c}\text { A-Wn 406.747-B.M } \\
(8)\end{array}$ & $\begin{array}{c}\text { A-Wn 406.747-B.M } \\
(9)\end{array}$ & - & - \\
\hline $\begin{array}{l}\text { La tempesta } \\
\text { de' dolori }\end{array}$ & $\begin{array}{l}\text { Donato } \\
\text { Cupeda? }\end{array}$ & 1703 & $\begin{array}{c}\text { A-Wn 406.747-B.M } \\
(18)\end{array}$ & - & - & - \\
\hline $\begin{array}{l}\text { II mistico } \\
\text { Giobbe }\end{array}$ & $\begin{array}{l}\text { Donato } \\
\text { Cupeda }\end{array}$ & 1704 & $\begin{array}{c}\text { A-Wn 406.747-B.M } \\
(24)\end{array}$ & $\begin{array}{c}\text { A-Wn 406.747-B.M } \\
(25)\end{array}$ & $\begin{array}{c}\text { A-Wn Mus.Hs. } \\
18852\end{array}$ & - \\
\hline $\begin{array}{l}\text { Le due pas- } \\
\text { sioni }\end{array}$ & $\begin{array}{l}\text { Pietro } \\
\text { Antonio } \\
\text { Bernardoni? }\end{array}$ & 1705 & $\begin{array}{c}\text { A-Wn 406.747-B.M } \\
(31)\end{array}$ & $\begin{array}{c}\text { A-Wn 406.747-B.M } \\
(32)\end{array}$ & $\begin{array}{c}\text { I-Vnm } \\
\text { MSS it. IV } \\
572.7\end{array}$ & - \\
\hline $\begin{array}{l}\text { La morte } \\
\text { vinta }\end{array}$ & $\begin{array}{l}\text { Pietro } \\
\text { Antonio } \\
\text { Bernardoni }\end{array}$ & 1706 & $\begin{array}{c}\text { A-Wn 406.745-B.M } \\
(5)\end{array}$ & $\begin{array}{c}\text { A-Wn 406.745-B.M } \\
(6)\end{array}$ & $\begin{array}{c}\text { A-Wn Mus. Hs. } \\
18751\end{array}$ & - \\
\hline $\begin{array}{l}\text { II sacrifizio } \\
\text { d'Isacco }\end{array}$ & $\begin{array}{l}\text { Pietro } \\
\text { Antonio } \\
\text { Bernardoni? }\end{array}$ & 1707 & $\begin{array}{c}\text { A-Wn 406.745-B.M } \\
(13)\end{array}$ & $\begin{array}{c}\text { A-Wn 406.745-B.M } \\
(14)\end{array}$ & $\begin{array}{c}\text { A-Wn Mus. Hs. } \\
19133\end{array}$ & - \\
\hline
\end{tabular}

3 Wenn nicht anders angegeben, finden sich die Quellen in der Musiksammlung der Österreichischen Nationalbibliothek (A-Wn). Insbesondere von den Libretti blieben weitere Exemplare mitunter auch in anderen Bibliotheken erhalten.

4 EVERETT, Paul. Opening „Il Sepolcro“: Ziani, Vivaldi and a question of stylistic authenticity. In Vivaldi vero e falso: Problemi di attribuzione. Studi di musica Veneta, Quaderni Vivaldiani VII. Florenz, 1992, S. 69-90.

5 Ist der Librettist mit einem Fragezeichen versehen, so wird er weder in den Libretti noch in der Partitur genannt. 


\begin{tabular}{|l|l|c|c|c|c|c|}
\hline Kurztitel & Librettist & Jahr & Libretto ita. & Libretto dt. & Partitur & Stimmen \\
\hline $\begin{array}{l}\text { La passione } \\
\text { nell orto }\end{array}$ & $\begin{array}{l}\text { Pietro } \\
\text { Antonio } \\
\text { Bernardoni }\end{array}$ & 1708 & $\begin{array}{c}\text { A-Wn 406.745-B.M } \\
(24)\end{array}$ & $\begin{array}{c}\text { A-Wn 406.745-B.M } \\
(25)\end{array}$ & $\begin{array}{c}\text { A-Wn Mus.Hs. } \\
19128\end{array}$ & - \\
\hline $\begin{array}{l}\text { Giesù flagel- } \\
\text { lato }\end{array}$ & $\begin{array}{l}\text { Pietro } \\
\text { Antonio } \\
\text { Bernardoni }\end{array}$ & 1709 & $\begin{array}{c}\text { A-Wn 406.745-B.M } \\
(32)\end{array}$ & $\begin{array}{c}\text { A-Wn 406.745-B.M } \\
(33)\end{array}$ & $\begin{array}{c}\text { A-Wn Mus.Hs. } \\
19129\end{array}$ & $\begin{array}{c}\text { A-Wn Mus. } \\
\text { Hs. } \\
19130\end{array}$ \\
\hline $\begin{array}{l}\text { La sapienza } \\
\text { umana }\end{array}$ & $\begin{array}{l}\text { Giovanni } \\
\text { Battista } \\
\text { Ancioni }\end{array}$ & 1710 & $\begin{array}{c}\text { I-Vnm } \\
\text { Dramm. } \\
0829.049\end{array}$ & - & $\begin{array}{c}\text { A-Wn Mus.Hs. } \\
18696\end{array}$ & - \\
\hline $\begin{array}{l}\text { Il sepolcro } \\
\text { nell' orto }\end{array}$ & $\begin{array}{l}\text { Silvio Stam- } \\
\text { piglia }\end{array}$ & 1711 & $\begin{array}{l}\text { I-Vnm } \\
\text { MISC }\end{array}$ & - & $\begin{array}{c}\text { A-Wn Mus. Hs. } \\
19131\end{array}$ & $\begin{array}{c}\text { A-Wn Mus. } \\
\text { Hs. }\end{array}$ \\
\hline
\end{tabular}

Tab. 1: Überblick über die Quellen zu Sepolcri von Marc`Antonio Ziani („Jahr“ bedeutet Aufführungsjahr)

\section{Die Libretti - Wandel in den Textbüchern}

Es wäre zu erwarten, dass Zianis Sepolcri - Partituren wie Libretti - im Wesentlichen sowohl stilistisch als auch strukturell übereinstimmen. Tatsächlich lassen sich jedoch vielfache Änderungen bzw. Entwicklungen feststellen, die sich bereits bei einem Vergleich der Libretti offenbaren.

1. Sowohl in den italienischen als auch in den deutschen Libretti der Sepolcri der Jahre 1701 und 1702 gibt der Librettist in den Fußnoten Quellennachweise. In der Regel handelt es sich dabei um Bibelstellen, aber auch auf Schriften der Heiligen Cyrill oder Hieronymus wird etwa in Il fascietto di Mirra (1701) verwiesen. Später finden sich keine Verweise mehr.

2. Wie in der Einleitung erwähnt änderte sich die Bezeichnung der Werke, bis 1705 lautet sie Rappresentazione Sacra, ab 1706 Oratorio. ${ }^{6}$ Als Beispiel sei die Titelseite von Zianis Sepolcro Le due Passioni (1705) wiedergegeben. ${ }^{7}$

6 SEIFERT, Herbert. Das Sepolcro - ein Spezifikum der kaiserlichen Hofkapelle. In Die Wiener Hofmusikkapelle III: Gibt es einen Stil der Hofmusikkapelle? Wien: Böhlau, 2011, S. 169.

$7 \quad$ A-Wn 406.747-B.M (31) (keine Foliierung) 


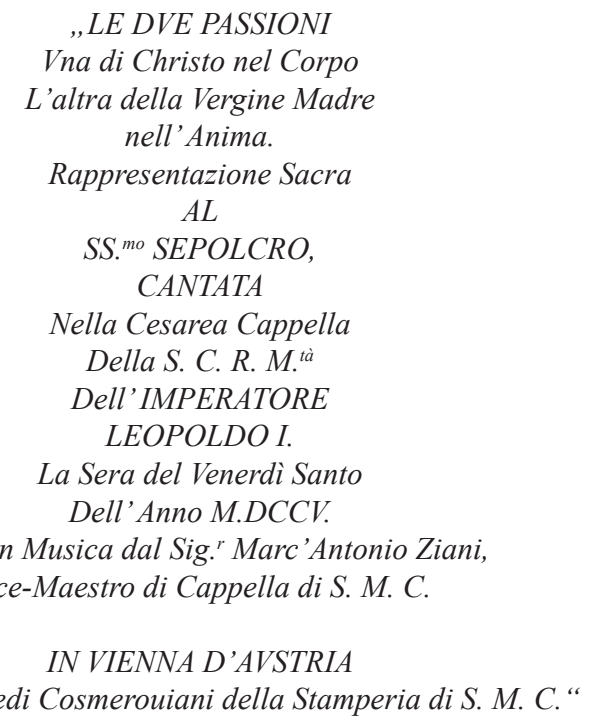

3. Ab 1705 fehlen jegliche Angaben zum Bühnenbild während bis 1704 unmittelbar nach der Auflistung der Figuren stets ein kurzer Text enthalten ist, der die Szenerie schildert, etwa in Il fascietto di Mirra (1701): ${ }^{8}$

„L'Apparato rappresenterà un Giardino di Gigli, e d'altri fiori. Nel mezo sarà una collinetta con un'Albero di MIRRA, presso il quale si uedrà la Sposa de'Cantici, che aurà nel seno un FASCIETTO di MIRRA, figura della Passione di christo, col motto: Fasciculus Myrrhae dilectus meus mihi; inter ubera mea commorabitur. Le saranno intorno diuerse Vergini di Gierusalemme in atto di presentarle fiori, e poma, espressiue della Passione. Di lontano sopra un Monticello si uedrà lo Sposo, ch'è figura di Christo, in atto di salire sopra una Palma, col motto: Ascendam in Palmam, \& apprehendam fructus ejus. “

Zwischen dem Text finden sich ebenfalls einzelne szenische Anweisungen, die sich allerdings darauf beschränken zu bemerken, welche Figur auf- bzw. abzutreten hat. Auch diese fehlen bereits ab 1705, also auch bei dem noch als Rappresentazione sacra benannten Sepolcro Le due Passioni.

4. Nach dem Titelblatt folgt in der Regel eine vermutlich vom Librettisten verfasste Einleitung. Wenn man davon absieht, dass diese 1702 fehlt, fällt auf, dass der Leser bis 1704 jeweils direkt angesprochen wird („Benigno Lettore“). $\mathrm{Ab} 1705$ fehlt diese persönliche Anrede, stattdessen folgt ab 1706 die sachliche Überschrift „Argomento“. In der Regel führt diese Einleitung in die Thematik des Stücks ein und stellt eventuell einen Zusammenhang mit der Passion her, wenn sich der Text nicht offensichtlich direkt damit befasst. Etwa vergleicht der Autor in der Einleitung von Il fascietto di Mirra (1701) das ,,Myhrren-Bueschlein“", das

\footnotetext{
$8 \quad$ A-Wn 406.742-B.M (30) (keine Foliierung)

9 Der Titel des deutschen Librettos lautet: Das Myrrhen-Bueschlein auff der Brust der Braut des Hohen-Lieds. A-Wn 406.742-B.M (31).
} 
die Braut des Hohen Lieds mit sich trägt, mit dem Leiden Christi, dessen Erinnerung der Leser ständig bei sich tragen sollte.

Erwähnenswert ist nicht zuletzt die Einführung von Giesù flagellato (1709), besteht sie doch nur aus einem einzigen Satz: Tunc ergo apprehendit Pilatus Jesum \& flagellavit. (Joan. 19.).

5. Im Laufe der Jahre verringerte sich die Zahl der im Sepolcro mitwirkenden Charaktere. 1701: acht, 1702 bis 1704 sieben, 1705 sechs, ab 1706 fünf.

Insbesondere die Änderungen der Bezeichnung und die fehlenden szenischen Anweisungen lassen Rückschlüsse auf eine Wandlung der Aufführungspraxis von einer szenischen Darstellung mit Bühnenbild und in Kostümen zur rein konzertanten Aufführung, vor allem in den Jahren 1705 und 1706 zu, die Komposition beeinflussen sie jedoch kaum.

Tatsächlich lässt sich in den Libretti auch ein Wandel in der Struktur der Texte bemerken, der sich stark auf die Vertonung auswirkt. Ab 1705 bilden die Libretti die textliche Grundlage für den für Opern oder Oratorien jener Zeit typischen Wechsel zwischen Rezitativen und Arien mit ein oder zwei Duetten und einem Chor am Ende. - Von 1701 bis 1704 ist dies jedoch nicht so. In den Libretti jener Zeit ist eine Vielzahl an Duetten und Terzetten zu finden, die sich oft nur über ein oder zwei Verse erstrecken und mit anderen Solisten zugewiesenen Versen in Zianis Vertonung zu musikalischen Nummern verbunden sind. ${ }^{10}$

\section{Die Partituren - Charakteristika von Zianis Sepolcri ${ }^{11}$}

Zianis Sepolcri sind wie auch die Sepolcri des 17. Jahrhunderts immer einsätzig und unterscheiden sich dadurch von den meisten Oratorien. ${ }^{12}$

Im Gegensatz zu Draghi, bei dessen Sepolcri mitunter keine Sinfonia notiert ist, ${ }^{13}$ weisen alle Sepolcri Zianis eine eröffnende Sinfonia auf. Diese folgt stets dem gleichen, zweiteiligen Aufbau. Der erste Satz ist in der Regel ein Grave oder Adagio, geprägt von Chromatik, Vorhalten und Modulationen. ${ }^{14}$ Mitunter findet sich am Ende dieses langsamen Satzes ein kurzes solistisches Zwischenspiel oder eine etwas schnellere Überleitung, die in den zweiten kontrastierenden Teil, die obligate Fuge mündet.

\footnotetext{
10 Siehe dazu unten.

11 Die Partitur des Sepolcro Le due Passioni (1705) stand dem Autor für diese Studie nicht zur Verfügung.

12 Es gibt allerdings auch einteilige Oratorien, etwa Le promesse nuzziali di S. Orsola (1702) von Carlo Agostino Badia, A-Wn 406.747-B (10). Dieses Werk wurde freilich im Ursulinenkloster aufgeführt.

13 Etwa: Antonio Draghi: La pietà contrastata (1673), A-Wn Mus.Hs. 16541 Mus. Leop.

14 Siehe Ex. 1.
} 
Zianis verwendet in diesen Fugen - wie auch in seiner liturgischen Musik zumeist mehrere kurze Themen, die er selbst soggetti nennt ${ }^{15}$ und meist in Engführung vorstellt.

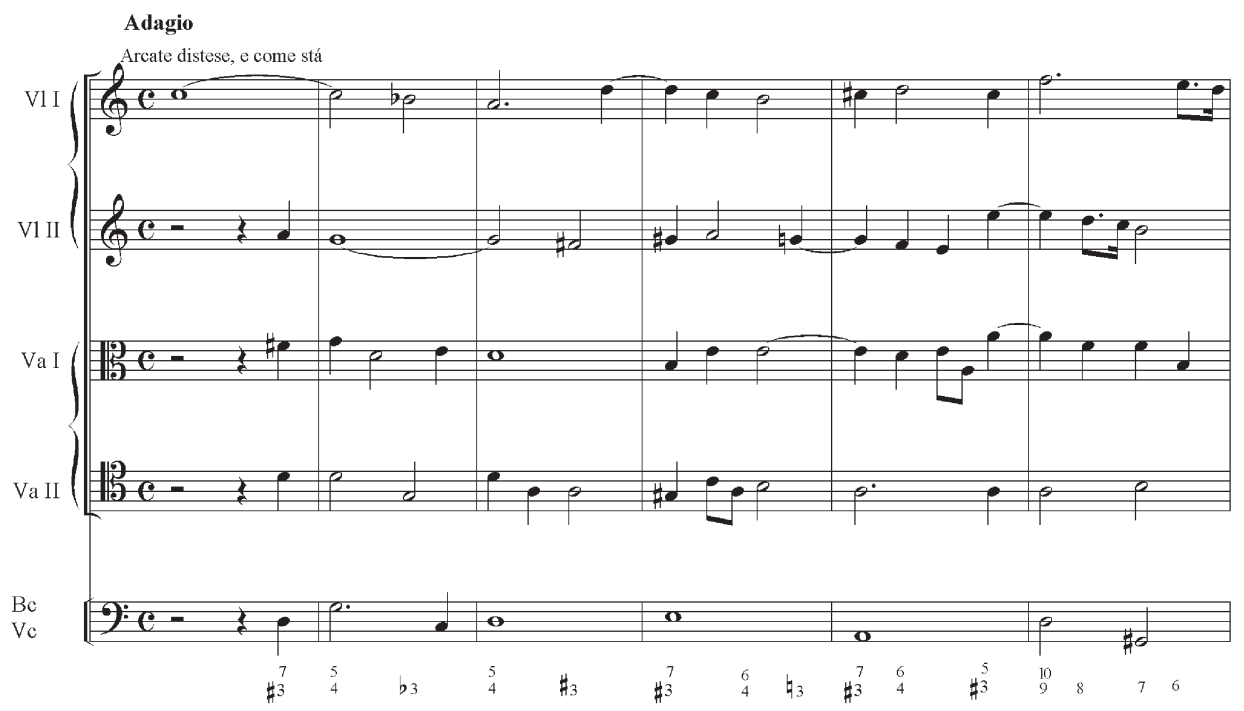

Ex. 1: La Passione nell'orto, A-Wn Mus.Hs.19128, fol 2v., Sinfonia

Treffend hat Paul Everett den ersten Satz des Sepolcro Le due Passioni (1705) als Verwendung einer Technik, die Musik zu zwingen, sich von einer Molltonart in die nächste zu winden, was eine Stimmung der Verwirrung und Unruhe provoziere, charakterisiert. ${ }^{16}$ - Angemerkt sei, dass Everett feststellen konnte, dass Antonio Vivaldi den Beginn dieser Sinfonia in fünf seiner Werke verwendete bzw. bearbeitete. ${ }^{17}$ Dies ist vor allem bemerkenswert, da Vivaldis Credo nur wenige Jahre nach Zianis Sepolcro entstand ${ }^{18}$ und man vermuten kann, das Sepolcro wäre lediglich einmal in Wien aufgeführt worden.

15 La morte vinta, A-Wn Mus.Hs.18751, fol. 3r. Siehe auch PROMINCZEL, Johannes. Die Kirchenmusik von Marc'Antonio Ziani: Quellen - Analyse - Werkverzeichnis (Dissertation). Wien, 2012, S. 175.

16 EVERETT, Paul. Ibd., S. 73: ,[...] the technique of forcing the music to meander rapidly from one minor key to another provokes an affection of perplexity and anxiety wholly appropriate, of course, to the scene at the sepulchre."

17 EVERETT, Paul. Ibd., S. 70: In den Werken Kyrie (RV 587), Credo (RV 591), Magnificat (RV 610a), Concerto madrigalesco (RV 129) sowie Konzert für Fagott (RV 491).

18 RYOM, Peter. Antonio Vivaldi: Thematisch-systematisches Verzeichnis seiner Werke (RV). 1. Ed. Wiesbaden, Leipzig \& Paris: Breitkopf \& Härtel 2007, S. 263. 
Zum Instrumentarium ist zu sagen, dass - wenig überraschend - Streicherbesetzungen mit Violinen und Violen in diversen Kombinationen und mit verschiedenen Schlüsseln die Sepolcri dominieren. Den Schlusschor von La morte vinta (1706) begleiten etwa vier Violen im Sopran- Alt-, Tenor- und Bassschlüssel. Häufiger werden vier Violen zweimal im Sopran- nebst Alt- und Tenorschlüssel notiert wie beispielsweise in der Sinfonia von La morte vinta. Es sei angemerkt, dass es durchaus möglich wäre, alle vier Stimmen mit Instrumenten zu spielen, deren Stimmung jeweils jener der heute üblichen (Alt-) Viola entspricht.

Auch die Kombination von Violen im Alt- und Tenorschlüssel mit zwei Violinen ist üblich. Bemerkenswert ist, dass in den späteren Sepolcri die Besetzung von zwei Violinen nebst im Altschlüssel notierter Viola vorherrscht, während etwa 1704 und 1706 Violen dominieren bzw. zumindest die hohen Streicher im Sopranschlüssel notiert werden. Erwähnenswert ist, dass das Violoncello separat neben dem Generalbass nahezu ausschließlich in den Einleitungssätzen notiert ist. Dort stimmt die Stimmführung jedoch mit dem Basso continuo überein. Solistisch wird das Violoncello nie eingesetzt.

Auch Gamben verwendet Ziani einige Male, allerdings fast ausschließlich in Il mistico Giobbe (1704). Sie treten stets paarweise auf, entweder zwei Altgamben oder Gamben im Alt- und Tenorschlüssel. Erwähnenswert ist, dass die Gamben, abgesehen vom Basso continuo, niemals gleichzeitig mit anderen Streichern spielen, sondern sich etwa mit den Violinen in ihren Einsätzen immer abwechseln. ${ }^{19}$

In den Werken von 1704 und 1706 bis 1708 setzt Ziani Bläser ein, allerdings eher selten. Entweder verdoppeln Zinken, Posaunen und Fagotte die Streicher bzw. verstärken den Generalbass oder Posaunen und Fagotte treten - mitunter durchaus virtuos - solistisch hervor. Am häufigsten verwendet Ziani Bläser in $L a$ morte vinta (1706), wo sie fest mit den Einsätzen des Demonio verbunden sind, dessen Arien sie begleiten. Erwähnenswert etwa die Auftrittsarie Hò già vinto, in welcher sich der Teufel über seinen vermeintlichen Sieg freut. ${ }^{20}$ In dieser mit Furioso bezeichneten Da capo-Arie verwendet Ziani die Bläser gleich auf beide Arten, einerseits verdoppeln die Zinken und Posaunen die vier Violen im Tutti, wobei sich das Orchester in der Regel auf Akkorde in statisch gleichförmigen Achteln beschränkt, die der Arie mitunter den Charakter eines AccompagnatoRezitativs verleihen. Andererseits treten Alt- und Tenorposaune auch solistisch hervor. Im Duett von Teufel und Tod Vil che sei obliegt die Begleitung neben dem Generalbass ausschließlich zwei teils virtuos geführten Fagotten. ${ }^{21}$

Die Assoziation von Bläsern beschränkt sich keineswegs auf das schlussendlich im Laufe des Sepolcro besiegte Böse, denn in La sacrifizio d'Isacco (1707)

19 Dies zeigt sich auch in der liturgischen Kirchenmusik. Vgl. PROMINCZEL, Johannes. Die Kirchenmusik von Marc'Antonio Ziani: Quellen - Analyse - Werkverzeichnis (Dissertation). Wien, 2012, S. 158.

21 La morte vinta, A-Wn Mus.Hs.18751, fol 43v. 
begleitet die Posaune in der Arie Non è giunta ancor l'età den Engel. ${ }^{22}$ Dies lässt jedenfalls auf eine sehr bewusste Verwendung der Bläser in Zianis Sepolcri schließen.

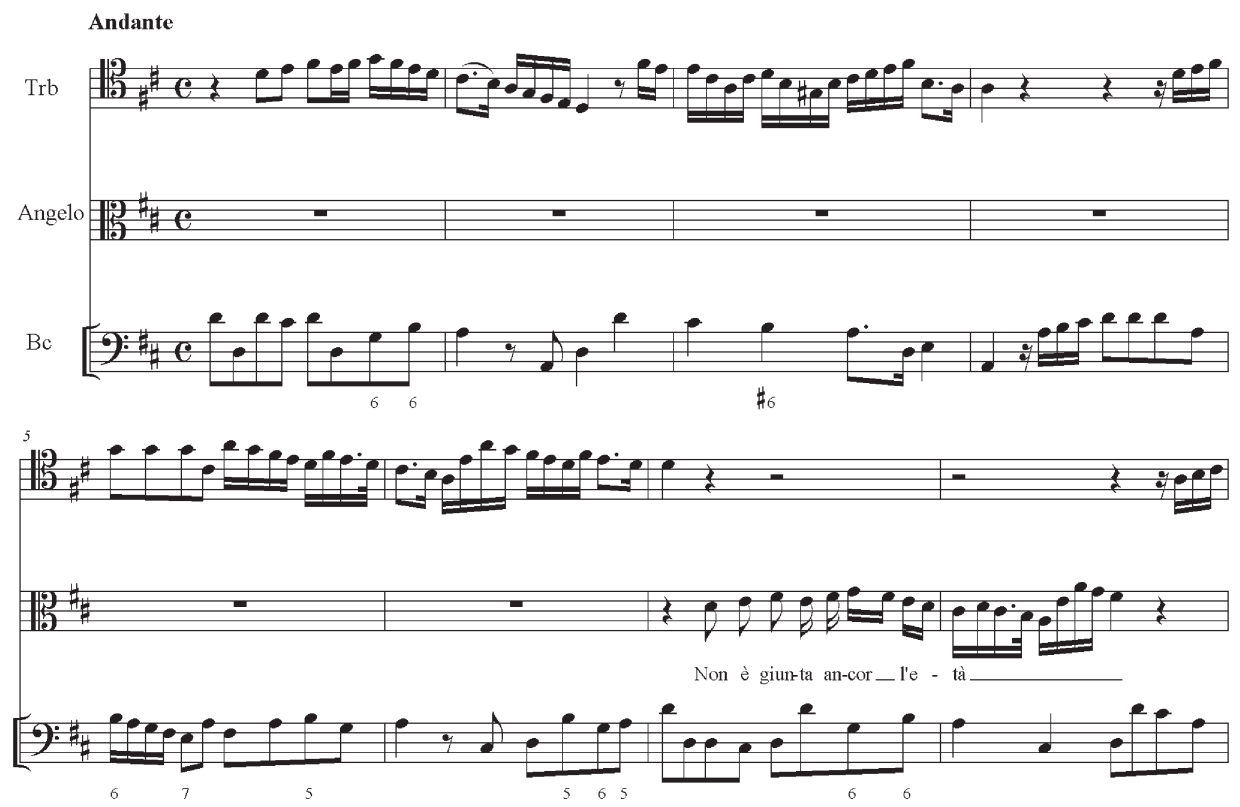

Ex. 2: Il sacrifizio d'Isacco, A-Wn Mus.Hs.19133, fol 61v., Arie des Angelo: Non è giunta l'età .

In den Ritornellen verwendet Ziani ausschließlich Violinen und Violen. Binnensinfonien wie bei Draghi sucht man bei ihm umsonst, allerdings ist ein Ritornell in Il mistico Giobbe (1704) 31 Takte lang, was ihm nahezu Binnensinfoniecharakter verleiht. ${ }^{23}$

Die Ritornelle beschließen größtenteils Arien, in denen der Sänger ausschließlich vom Basso continuo begleitet wird. Thematisch-melodisch hängt das Ritornell stets mit der jeweilige Arie zusammen, zumindest das Eingangsmotiv stimmt überein. Mitunter sind Ritornelle auch insofern mit den Arien verknüpft, als die Streicher des Ritornells bereits im letzten Takt der Arie einsetzen. ${ }^{24}$

In den sieben untersuchten Partituren steht nur vier Mal ein Ritornell zwischen Rezitativ und Arie um nach der Arie wiederholt zu werden. Umgekehrt gibt es

22 Siehe Ex. 2.

23 Il mistico Giobbe, A-Wn Mus.Hs.18852, fol 31r.

24 Etwa am Ende der Aufrittsarie des Giusto tribulato in Il mistico Giobbe, A-Wn Mus.Hs. 18852 fol. 8 r. 
auch nur wenige ausschließlich vom Basso continuo begleitete Arien, auf die kein Rezitativ folgt, beispielsweise die Arie Si fransero pietosi der Maria Giacobbe aus Il sepolcro nell'orto (1711), die mit dem punktierten Rhythmus im langsamen 12/8-Takt an den Siciliano erinnert. ${ }^{25}$

Grundsätzlich sind jene Arien mit Instrumentalbegleitung etwas weniger häufig, als jene die nur vom Basso continuo begleitet werden. In Il sacrifizio d'Isacco (1707) sind von 14 Arien acht nur vom Basso continuo begleitet, in La sapienza umana (1710) von 18 Arien elf, in Il sepolcro nell'orto (1711) von 13 Arien neun.

Spätestens ab 1706, möglicherweise auch schon 1705, dominiert eindeutig die Da capo-Form, die Ziani auch in Duetten einsetzt, während 1704 und wie die Libretti vermuten lassen auch davor, andere Arienformen vorherrschen, zumeist handelt es sich dabei um Abwandlungen von Strophenarien. Die unveränderte melodisch-harmonische Wiederholung der ersten Strophe ist nur einmal zu finden, allerdings singt die erste Strophe mit Amor Divino ein Tenor, die zweite mit der Consolazione ein Sopran. ${ }^{26}$ Deutlich häufiger werden die Wiederholungen variiert oder es wird gar nur der Themenkopf übernommen auf den neues melodisches Material folgt. Ein Beispiel für den Aufbau eines Duetts der Hartnäckigkeit (L'Ostinazione, Sopran) und des beglückten Gottlosen (L'Empio prosperato, Tenor) in Il mistico Giobbe (1704) ist Nobil core è più saldo.

$\begin{array}{ccccccc}\text { Text } & \mathrm{A} & \mathrm{B} & \mathrm{A} & \mathrm{A}^{\prime} & \mathrm{B}^{\prime} & \mathrm{C} \\ \text { Musik } & 1 & 2 & 1 & 3 & 4 & 1 \\ \text { Sänger } & \text { Ostinazione } & \text { Ostinazione } & \text { Ostinazione } & \text { Empio prosp. } & \text { Empio prosp } & \begin{array}{c}\text { Ostinazione } \\ \text { Empio prosp. }\end{array}\end{array}$

Tab. 2: Struktur des Duetts Nobil core è più saldo aus Il mistico Giobbe, A-Wn Mus.Hs.18852, fol $71 \mathrm{r}$.

A' und B' unterscheiden sich insofern von A und B, als sie geringfügig verändert sind, etwa wurden einzelne Takte eingefügt oder gestrichen, auch rhythmische Variationen sind zu bemerken. C erinnert thematisch an A, wird aber schlussendlich doch gänzlich anders geführt.

Bemerkenswert ist auch der Beginn von Il mistico Giobbe. Nach der Sinfonia tritt der ,gequälte Gerechte", gemeint ist Ijob, auf, der sein Schicksal beklagt. ${ }^{27}$ Er beginnt seine Arie mit der Frage „Fra tante mie pene, deh chi me consola?“" Nach dem Ritornell und einem Rezitativ stellt er - auch melodisch und harmonisch gleich - noch einmal dieselbe Frage, erhält auf dieses kurze Zitat aus der Auftrittsarie in der Form des Rezitativs von Innocenza, Amor Divino und Pazienza, Antwort, dass sie ihm zur Seite stehen, was sie im darauf folgenden Terzett auch bekräftigen.

\footnotetext{
25 Il sepolcro nell'orto, A-Wn Mus.Hs.19131, fol 28r.

26 Il mistico Giobbe, A-Wn Mus.Hs.18852, fol. 38v.

27 Il mistico Giobbe, A-Wn Mus.Hs.18852; Eröffnungsarie ab fol 7r., Ritornell fol. 8r., Rezitativ fol $8 \mathrm{v}$., Terzett fol 10r.
} 
Generell ist in Il Mistico Giobbe (1704) eine Reihe an Duetten und Terzetten oder größeren Ensembles zu finden. Ähnliches zeigt sich auch in den Libretti von 1701 bis 1703. In späteren Sepolcri sind Duette eher selten, höchstens ein oder zwei, größere Ensembles und auch die Kombination von verschiedenen Besetzungen gibt es nur am Ende der Komposition.

Ziani hat drei unterschiedliche Formen des Schlusssatzes:

1. Eine Chorfuge (1707 und 1713).

2. Ein Solist stellt die Fugenthemen hintereinander vor, der Basso continuo bringt die Kontrasubjekte, ein zweiter Solist variiert anschließend die Themen, dann folgt die Chorfuge (1704, 1708 und 1709).

3. Eine Chorfuge, dann folgt ein kurzes Duett von Sopran und Alt, anschlieBend wird die Fuge wiederholt (1706 und 1710).

Angemerkt sei, dass die Streicher mit Ausnahme der Bassinstrumente in den Chorfugen der Sepolcri niemals colla parte geführt werden.

Arietten und ariose Abschnitte, vor allem am Ende von Rezitativen sind in Il mistico Giobbe anzutreffen. Accompagnato-Rezitative finden sich hingegen ausschließlich zwei Mal in La sapienza umana (1710), wobei es sich eigentlich nur um Accompagnato-Abschnitte in Secco-Rezitativen handelt, denn die Singstimme wird jeweils nur am Beginn des jeweiligen Rezitativs durch die Streicher begleitet. $^{28}$

Abschließend sei angemerkt, dass es scheint, als hätte der Anlass kaum Einfluss auf Zianis Kompositionsstil. Obwohl die Sepolcri in der Hofmusikkapelle am Karfreitag unmittelbar nach Öffnung des Grabs aufgeführt wurden ${ }^{29}$ verzichtet er weder auf die vor allem am Ende des ersten Teils der Da capo-Arien zu findenden teils virtuosen Koloraturen noch auf den Einsatz von Affekten. Ziani beweist geradezu Humor, als er in der Arie Hò già vinto des Demonio in La morte è vinta (1706) ausgerechnet beim Text Non più sento (gran tormento) die Instrumente pausieren lässt und den Demonio und den Basso continuo im Unisono führt. ${ }^{30}$ Erwähnenswert ist auch die mit La Scala betitelte Arie des Servo in Il sacrifizio d'Isacco (1707). ${ }^{31}$ Sowohl in der Instrumentalbegleitung als auch in der Singstimme setzt Ziani eine sechsstufige Tonleiter in punktierten halben Noten ein, wobei diese Reihe von do bis la jeweils in der Partitur eigens gekennzeichnet ist. Bereits ohne Da capo findet man diese Tonleiter insgesamt $22 \mathrm{Mal}$ in der Arie, acht Mal davon gespiegelt.

28 La sapienza umana, A-Wn Mus.Hs.18696, fol 5r. und 15r.

29 Im Libretto von Giesù flagellato ist zu lesen „cantato nell' August. Capella“ (A-Wn 406.745B.M (32), in der Partitur jedoch „cantato all' SS. Sepolcro [...] venerdi santo“ (A-Wn Mus. Hs.19129). Daraus ist zu schließen, dass das Werk zumindest ursprünglich für den Karfreitag gedacht war.

30 La morte è vinta, A-Wn Mus.Hs.18751, fol 10v.

31 Il sacrifzio d'Isacco, A-Wn Mus.Hs.19133, fol 36v. 


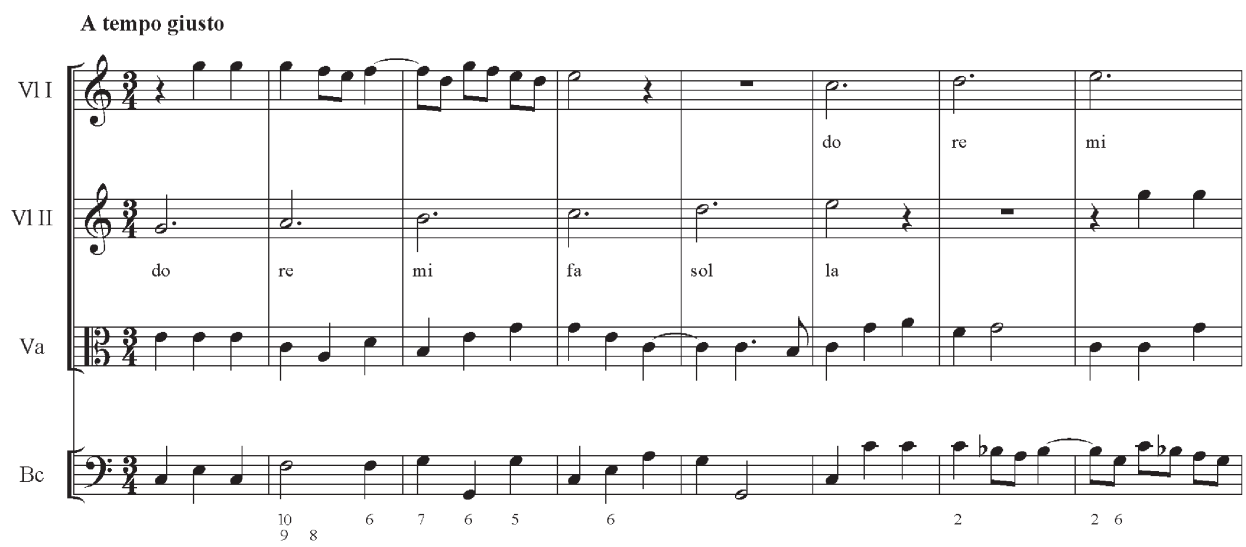

Ex. 3: Il sacrifizio d'Isacco, A-Wn Mus.Hs.19133, fol 36v., Arie des Servo: Quando il ciel minaccia „La Scala“, Instrumentale Einleitung.

\section{Conclusio}

Zusammenfassend ist festzustellen, dass der Wandel des Sepolcro in jenen Jahren, in denen sich Ziani dieser Gattung widmete, schrittweise erfolgt ist. Erwähnenswert ist insbesondere die strukturelle Veränderung des Librettos, die die Komposition stark beeinflusste. Während ab 1705 Arien dominieren, finden sich in der Zeit davor zahlreiche Ensembles, auch freiere Arienformen. Dies ist wohl in erster Linie mit dem Wechsel des Hofpoeten zu begründen. Denn Donato $\mathrm{Cu}$ peda, der wohl die Libretti für Zianis erste vier Sepolcri verfasst hatte, starb 1704.

Worauf der Wegfall der szenischen Anweisung 1705 und die im Jahr darauf resultierende Änderung der Gattungsbezeichnung auf den Titelblättern der Libretti zurückzuführen ist, konnte nicht geklärt werden. Festzuhalten ist jedenfalls, dass die fehlende Beschreibung der Szenerie nicht auf Josef I. zurückzuführen sein dürfte, da dies noch zu Lebzeiten Kaiser Leopold I. geschah.

Johannes Prominczel (jopromi@yahoo.de).

\section{ABSTRACT \\ THE SEPOLCRI OF MARC'ANTONIO ZIANI}

During his engagement at the Imperial Court between 1701 and 1711 Marc'Antonio Ziani composed one "Sepolcro“ every year. A Sepolcro is a sacred dramatic composition performed at the court chapel in front of the holy grave at Good Friday. This paper tries to find out if this genre changed at that time and if Ziani brought it closer to the Oratorio. As a matter of fact the structural transformation of the libretti caused more Da capo- Arias in the later years whereas in previous years we find Duetti and Choirs more frequently. 


\section{Key words}

Marc'Antonio Ziani, Antonio Draghi, Sepolcro, Oratorium, Rappresenatzione sacra, Imperial court, court chapel, Wiener Hofmusikkapelle, fugue, sinfonia, church music, Leopold I., Joseph I, Karl VI., libretto, Nicolò Minato, Donato Cupeda, Pietro Antonio Bernardoni

\section{Bibliography}

BREUER, Sybille. Johann Heinrich Schmelzers „Le memorie dolorose“ 1678 und das Wiener Sepolcro (Diplomarbeit). Wien, 1999.

GRUBER, Gernot. Das Wiener Sepolcro und Johann Joseph Fux: Nach einem Vortrag gehalten vor der Jahreshauptversammlung der Johann-Joseph-Fux-Gesellschaft am 3. Dezember 1968. Graz, 1972

EVERETT, Paul. Opening ,il Seplcro“: Ziani, Vivaldi and a question of stylistic authenticity. In Vivaldi vero o falso: Studi di attribuzione. Studi di musica Veneta, Quaderni Vivaldiani VII. Florenz, 1992, S. 69-90.

PROMINCZEL, Johannes. Die Kirchenmusik von Marc'Antonio Ziani: Quellen - Analyse - Werkverzeichnis (Dissertation). Wien, 2012.

RENKER, Gustav. Das Wiener Sepolcro (Dissertation). Wien, 1913.

RYOM, Peter. Antonio Vivaldi: Thematisch-systemaitsches Verzeichnis seiner Werke (RV). 1. Ed. Wiesbaden, Leipzig \& Paris: Breitkopf \& Härtel 200.

SAUNDERS, Steven. The Antecedents of the Viennese Sepolcro. In Relazioni musicali tra Italia e Germania nell' età barocca. Atti del VI Convegno internazionale sulla musica italiana nei secoli XVII-XVIII, 11. - 13. Juli 1995, Como: 1997, S. 63-83.

SCHNITZLER Rudolf. The sacred dramatic music of Antonio Draghi (Dissertation). Chapel Hill: 1971.

SCHNITZLER, Rudolf. The Viennese Oratorio and the work of Ludovico Ottavio Burnacini. In L'opera italiana a Vienna prima di Metastasio, Firenze, 1990, S. 217-237.

SEIFERT, Herbert. Das Sepolcro - ein Spezifikum der kaiserlichen Hofkapelle. In Die Wiener Hofmusikkapelle III: Gibt es einen Stil der Hofmusikkapelle?. Wien: Böhlau, 2011, S. 163-173.

TRUMMER, Johann. Sepolcro-Kompositionen und ihre frömmigkeitsgeschichtliche Bedeutung. In Bericht des wissenschaftlichen Symposions auf Schloss Seggau 14. - 16. Oktober 2005 anlässlich des Jubiläums , ,50 Jahre Johann-Joseph-Fux-Gesellschaft“. Tutzing, 2008, S. 117-128.

\section{Libretto collectible prints}

A-Wn 406.742-B.M

A-Wn 406.745-B.M

A-Wn 406.747-B.M

\section{Scores (Manuscripts)}

ZIANI, Marc'Antonio. Il mistico Giobbe, A-Wn Mus.Hs.18852

ZIANI, Marc'Antonio. La morte vinta, A-Wn Mus.Hs.18751

ZIANI, Marc'Antonio. Il sacrifizio d'Isacco, A-Wn Mus.Hs.19133

ZIANI, Marc'Antonio. La passione nell'orto, A-Wn Mus.Hs.19128

ZIANI, Marc'Antonio. Giesù flagellato, A-Wn Mus.Hs.19129, Stimmen A-Wn Mus.Hs.19130

ZIANI, Marc'Antonio. La sapienza umana, A-Wn Mus.Hs.18696

ZIANI, Marc'Antonio. Il sepolcro nell'orto, A-Wn Mus.Hs.19131; Stimmen A-Wn Mus.Hs. 19132 